\title{
Crystal Interfaces
}

Cite as: Journal of Applied Physics 35, 234 (1964); https://doi.org/10.1063/1.1713075 Submitted: 11 July 1963 . Published Online: 20 July 2004

N. H. Fletcher

\section{ARTICLES YOU MAY BE INTERESTED IN}

Crystal Interfaces. Part I. Semi-Infinite Crystals

Journal of Applied Physics 34, 117 (1963); https://doi.org/10.1063/1.1729050

Crystal Interfaces. Part II. Finite Overgrowths

Journal of Applied Physics 34, 123 (1963); https://doi.org/10.1063/1.1729051

Size Effect in Heterogeneous Nucleation

The Journal of Chemical Physics 29, 572 (1958); https://doi.org/10.1063/1.1744540

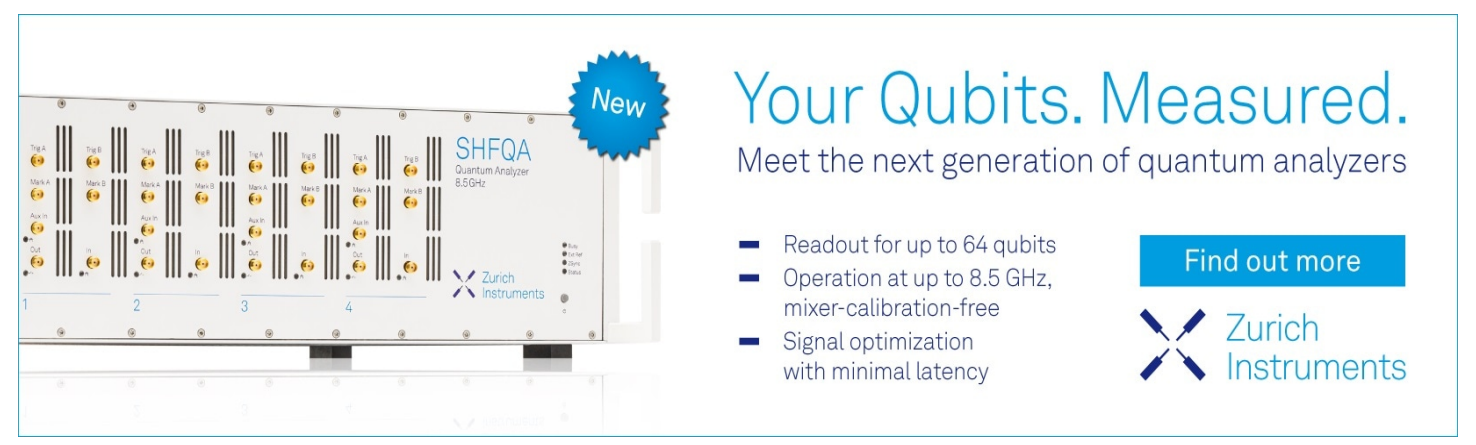


mum, is given by

$$
d_{N} / \theta^{-1}=\left(8 \cosh ^{-1} \sqrt{3} / 3^{5 / 4}\right)(1-\tau)^{-1} .
$$

We have here also that the normalized activation energy and the normalized width are functions only of $\tau$, the normalized stress; $U_{N} / E_{0}$ and $d_{N} / \theta^{-1}$ are plotted in Figs. 12 and 13 , respectively.

(3) Fisher. The formula for the activation energy $U_{F}$ derived by Fisher can be written

where

$$
U_{F}=\left(\mu^{2} / \sigma b\right) f(R)
$$

$$
f(R)=\cos ^{-1} R-R\left(1-R^{2}\right)^{\frac{1}{2}},
$$

and

$$
R=1-U_{0} / \mu b .
$$

In general $R \simeq 1$, and we can write

$$
f(R) \simeq\left(\frac{2}{3}\right)[2(1-R)]^{\frac{3}{2}}
$$

and, therefore,

$$
U_{F} / E_{0} \simeq 2 / \tau
$$

This last equation is exactly what is obtained at low stresses using the triangle force and taking $p=\frac{1}{3}$ [cf. Eq. (4.8)]. In Fisher's model the activation half-width (corresponding to $x_{p}$ ) is $d_{F}$, the half-width of line to be unpinned. This can be written as

$$
d_{F} / \theta^{-1}=(\mu \theta / \sigma b)\left[\left(U_{0} / \mu b\right)\left(2-U_{0} / \mu b\right)\right]^{\frac{1}{2}} \simeq 3 / \tau .
$$

This is the same result [cf. Eq. (4.6)] obtained at low stresses for $\theta x_{p}$ in the triangle force approximation with $p=\frac{1}{3}$.

\title{
Crystal Interfaces*
}

\author{
N. H. FLETChER \\ Department of Physics, The University of New England, Armidale, N. S. W., Australia
}

(Received 11 July 1963; in final form 21 August 1963)

\begin{abstract}
An expression for the energy of an interface of general form between two crystals of arbitrary structures and relative orientations is derived in a form suitable for a variational calculation. This variational approach is applied to a one-dimensional interface between two two-dimensional crystals of differing lattice constants. In general there is a minimum in the interfacial energy when the lattice distances in the two crystals are in the ratio of small integers. Particular cases in which the surface potential is either sinusoidal or parabolic are discussed and detailed curves of interfacial energy as a function of lattice misfit are calculatcd.
\end{abstract}

\section{INTRODUCTION}

$\mathrm{F}^{\mathrm{o}}$ R many problems in crystal physics a detailed knowledge of the energy of the interface between two crystalline phases is of great importance. Consideration of the most general type of interface is very complicated because of the variety of crystal structures and relative orientations. ${ }^{1}$ It is possible, however, to go some way in the formulation of a general theory of interfacial energy, though resort must be had either to numerical methods or to the consideration of specially simple cases if explicit results are required.

The energy of crystal interfaces has been discussed recently by van der Merwe, ${ }^{2}$ extending earlier work by the same author. ${ }^{3} \mathrm{His}$ approach was to consider first the elastic stresses at the boundary of two two-dimensional crystals meeting at a one-dimensional boundary, for

\footnotetext{
* This work is supported by the Australian C.S.I.R.O., and by the Atmospheric Science Program of the U. S. National Science Foundation under Grant G19677.

${ }^{1}$ S. Amelinckx and W. Dekeyser in Solid State Physics, edited by F. Seitz and D. Turnbull (Academic Press, Inc., New York, 1959), Vol. 8, p. 327.

${ }^{2}$ J. H. van der Merwe, J. Appl. Phys. 34, 117, 123 (1963).

3 J. H. van der Merwe, Proc. Phys. Soc. (London) A63, 616 (1950).
}

the case of small misfits, and then to extend the analysis to three-dimensional crystals by considering the array of dislocations at the interface. The mathematical model used appears to give elegant and accurate results but, as we shall see later, their range of applicability is confined to quite small misfits between the two lattices.

In many physical problems involving molecular solids the range of lattice parameters may be great and it is of interest to have estimates of interfacial energies over wide misfit ranges and particularly for simple lattice parameter ratios like 2:1 which one might expect to correspond to minima in the misfit energy.

It is the purpose of this paper to discuss the interfacial energy for some simple cases using a mathematical treatment, rather different from that of van der Merwe, which is better able to treat cases where the misfit between the two crystal lattices is large.

\section{GENERAL INTERFACES}

Consider a plane, low-index face of a simple crystal upon which a single atom or molecule of another 
substance is adsorbed at zero absolute temperature. The potential energy of this molecule at a point $\mathbf{r}$ can be expressed as a two-dimensional Fourier series

$$
V(\mathbf{r})=\sum_{\mathbf{k}} V_{\mathbf{k}} e^{i \mathbf{k} \cdot \mathbf{r}},
$$

where the vectors $\mathbf{k}$ belong to the reciprocal lattice of the surface.

Now suppose that instead of a single adsorbed molecule, a semi-infinite crystal with a plane low-index face is brought into contact with the crystalline substrate. Ignoring for the moment any possible elastic distortions of the two structures near the interface, the total interface energy from the point of view of the overgrowing crystal can be written.

$$
E_{1}=\sum_{\mathbf{r}_{0}} \sum_{\mathbf{k}} V_{\mathrm{k}} e^{i \mathbf{k} \cdot \mathrm{r}_{0}}
$$

where the $\mathbf{r}_{0}$ are the undisturbed molecular positions at the interface. Equation (2) represents an approximation in that possible displacements normal to the surface have been neglected in defining the Fourier coefficients $V_{\mathrm{k}}$ in (1). This can be remedied by a slight change in the definition, which is not important. More importantly however we have neglected the effect of possible molecular orientation constraints in the crystal. This may easily give $r$ values very different from those found with a single molecule, free to rotate, and must be taken account of in determining the $V_{\mathrm{k}}$.

The physical situation is now, however, quite symmetrical between the substrate and the overgrowth so that the interface energy from the viewpoint of the substrate molecules is

$$
E_{2}=\sum_{\mathbf{r}_{0}^{\prime}} \sum_{\mathbf{k}^{\prime}} V_{\mathbf{k}^{\prime}} e^{i \mathbf{k}^{\prime} \cdot \mathbf{r}^{\prime}},
$$

where $\mathbf{r}_{0}{ }^{\prime}$ are the molecular positions in the substrate and $\mathbf{k}^{\prime}$ are the reciprocal surface lattice vectors of the overgrowth. The total interface energy is clearly $\frac{1}{2}\left(E_{1}+E_{2}\right)$ since all interactions are counted twice if (2) and (3) are summed.

In the most general interface problem we have two crystals of differing structure and with arbitrary relative orientations at the interface. For our treatment it is necessary to impose some restriction on the relative orientations and lattice parameters of the two crystals so that there exists, in the interface, a two-dimensional superlattice of atomic positions common, except for a possible small translation, to the substrate and the overgrowth. For two crystals of arbitrary structure and orientation such a superlattice can be generated as follows. The interface plane of each crystal has a simple two-dimensional lattice, and, by a simple translation, a lattice point of one lattice can be brought into coincidence with a point of the other. In general there are no other coincident lattice points but, in a large enough area of interface, there are always pairs of lattice points arbitrarily close to coincidence. Two pairs of points can then be brought to coincidence by a very small rotation or a very small change in the lattice parameters of one crystal, or both, and by continuity the effect on the interface energy can be made arbitrarily small. The vectors joining the two pairs of coinciding lattice points to the origin are now vectors of the superlattice and the adjustment which brought these pairs of points to coincidence has at the same time created coincidences at all other points of the superlattice.

This artifice excludes no physically important possibilities. For a one-dimensional interface, for example, it merely requires that the lattice parameters of the two crystals be in rational rather than irrational ratio. Mathematically however it reduces the number of possible cases from a nondenumerable to a denumerable infinity and allows the use of Fourier series instead of Fourier integrals.

The possibility of elastic strain of the two lattices may now be admitted, since in this way it may be possible to lower the misfit energy. Whatever these strains they must be periodic in the superlattice, so that they can be expressed as the sum of two Fourier series in terms of $\mathbf{K}$, the reciprocal vectors of the superlattice. One of these series represents displacements in the direction of the corresponding $\mathbf{K}$ and the other takes account of displacement perpendicular to it but still in the plane of the interface.

Since stresses across the interface must vanish, the elastic distortions must be in inverse ratio to some elastic modulus $\lambda$ of the crystal on either side of the interface, and of opposite sign. Thus a molecule of the overlay initially at position $\mathbf{r}_{0}$ is displaced to

$$
\mathbf{r}=\mathbf{r}_{0}+\frac{1}{\lambda} \sum_{\mathbf{K}} \mathbf{D}_{\mathbf{K}} e^{i \mathrm{~K} \cdot \mathrm{r}_{0}}+\frac{1}{\lambda}-\sum_{\mathbf{K}} \mathbf{D}_{\mathbf{K}} e^{i \mathrm{~K} \cdot \mathrm{r}_{0}},
$$

where $\lambda$ is the appropriate elastic modulus of the overlay and $\mathbf{D}_{\mathbf{K}}, \mathbf{D}_{\mathbf{K}}{ }^{\prime}$ are displacement vectors, respectively parallel and perpendicular to $\mathbf{K}$. Note that the term $\mathbf{K}=0$ is to be included in the summation as corresponding to uniform displacement without distortion. In an exactly similar way we have, for the substrate,

$$
\mathbf{r}^{\prime}=\mathbf{r}_{0}{ }^{\prime}-\frac{1}{\lambda^{\prime}} \sum_{\mathbf{K}} \mathbf{D}_{\mathbf{K}} e^{i \mathbf{K} \cdot \mathbf{r}_{0}{ }^{\prime}}-\frac{1}{\lambda^{\prime}} \sum_{\mathbf{K}} \mathbf{D}_{\mathbf{K}^{\prime}} e^{i \mathbf{K} \cdot \mathbf{r}_{0}{ }^{\prime}}
$$

Now in Eqs. (2) and (3) not only have the molecular coordinates been displaced, but also those of the potential, and these have additive effects. Thus (2) becomes

$$
\begin{aligned}
E_{1}=\sum_{\mathbf{r}_{0}} \sum_{\mathbf{k}} V_{\mathbf{k}} \exp i \mathbf{k} \cdot\left[\mathbf{r}_{0}+\left(\frac{1}{-}+\frac{1}{\lambda}\right)\right. & \\
& \left.\times \sum_{\mathbf{K}}\left(\mathbf{D}_{\mathbf{K}}+\mathbf{D}_{\mathbf{K}}{ }^{\prime}\right) e^{i \mathbf{K} \cdot \mathbf{r}_{0}}\right],
\end{aligned}
$$


while (3) becomes

$$
\begin{aligned}
& E_{2}=\sum_{\mathbf{r}_{0}^{\prime}} \sum_{\mathbf{k}^{\prime}} V_{\mathbf{k}^{\prime}} \exp ^{\prime} \mathbf{k}^{\prime} \cdot\left[\mathbf{r}_{0}^{\prime}-\left(\frac{1}{\lambda}+\frac{1}{\lambda^{\prime}}\right)\right. \\
& \left.\times \sum_{\mathbf{K}}\left(\mathbf{D}_{\mathbf{K}}+\mathbf{D}_{\mathbf{K}}{ }^{\prime}\right) e^{i \mathbf{K} \cdot \mathbf{r}^{\prime}}\right] \text {. }
\end{aligned}
$$

In both (6) and (7) the summations over $\mathbf{r}_{0}$ and $\mathbf{r}_{0}{ }^{\prime}$ may conveniently extend over one cell of the superlattice with appropriate fractional contributions from molecules on edges or corners. Both $E_{1}$ and $E_{2}$ can then be divided by the area $S$ of this cell so that the interface mistit energy per unit area is

$$
E_{m}=\left(E_{1}+E_{2}\right) / 2 S .
$$

Since, however, the crystals are distorted at the interface there is an elastic strain energy which must be included in the total interface energy. If both substrate and overlay are semi-infinite slabs then it is shown in standard texts on elasticity that periodic elastic stresses and strains fall off exponentially away from the free surface in the same way as the displacements, that is,

$$
\mathbf{D}_{\mathrm{K}}(z)=\mathbf{D}_{\mathbf{K}}(0) \exp (-K z)
$$

where $z$ is distance from the interface and $K$ is the magnitude of $\mathbf{K}$.

Thus, if $x$ is measured parallel to $\mathbf{K}$, the strain energy in the semi-infinite overlay due to the mode $\mathbf{D}_{\mathbf{K}}$ is obtained by integrating half the product of stress and strain throughout the volume involved, which yields, per unit area of interface,

$$
\begin{aligned}
\frac{K}{4 \pi} \int_{0}^{\infty} \int_{0}^{2 \pi / K} \lambda^{-1} K^{2} D_{\mathrm{K}^{2}} \cos ^{2} K x & \\
\cdot \exp (-2 K z) d x d z & =\frac{K}{8 \lambda} D_{\mathrm{K}^{2}},
\end{aligned}
$$

so that the total elastic strain energy per unit area is

$$
E_{e}=\frac{1}{8}\left(\frac{1}{\lambda}+\frac{1}{\lambda^{\prime}}\right) \sum_{\mathbf{K}} K\left(D_{\mathbf{K}^{2}}+D_{\mathbf{K}^{\prime 2}}\right)
$$

The complete expression for the interfacial energy is thus

$$
E=E_{m}+E_{e}
$$

which can be written in detail using (6), (7), (8), and (11).

This expression (12) gives the interfacial energy in a form suitable for a variational calculation. It is clear that for the equilibrium state of the interface the energy $E$ is a minimum under variation of the parameters $\mathbf{D}_{\mathbf{K}}$, and this may be made a basis for the evaluation of $E$ by either a numerical or a quasianalytical process.

\section{ONE-DIMENSIONAL INTERFACES}

The variational expression (12) is sufficiently general to allow the calculation of the energy of any interface once the surface potentials are known. This is, however, a rather laborious calculation for a general case and best performed by numerical methods.

To illustrate the use of this variational approach and show the sort of detailed information which can be drawn from it we apply it to consideration of the simple case of the energy of the one-dimensional interface between two two-dimensional crystals of differing lattice parameters. For mathematical simplicity we further suppose that the crystals consist of atoms of one type or of symmetrical molecules so that the surface potential can be expanded in a cosine series. Inclusion of antisymmetric terms would not be expected to modify the results in general form, though they would, of course, introduce quantitative changes.

Let the substrate have lattice spacing $a$ and the overlay $b$, along the direction of the interface, and suppose that

$$
N a=M b,
$$

where $N$ and $M$ are integers with no common factor. Equation (13) then defines the superlattice, and the various lattice and reciprocal lattice vectors are simply the numbers

$$
\begin{aligned}
& r_{0}=\left(m+\frac{1}{2}\right) b ; \quad r_{0}^{\prime}=\left(m+\frac{1}{2}\right) a ; \\
& k=2 \pi n / a ; \quad k^{\prime}=2 \pi n / b ; \quad K=2 \pi s / N a,
\end{aligned}
$$

where $m, n$, and $s$ are integers. The half-integral displacements in $r_{0}$ and $r_{0}{ }^{\prime}$ correspond to the physical situation in which the potential minima are midway between the atoms, as in a close-packed structure.

In this two-dimensional problem clearly only the parallel displacements $D_{\mathbf{K}}$ occur, and the total interface energy per unit length can be written

$$
\begin{aligned}
E=\frac{1}{2 M b} \sum_{m=1}^{M} \sum_{n} V_{n} \cos \left\{\frac { 2 \pi n } { a } \left[\left(m+\frac{1}{2}\right) b\right.\right. & \left.\left.+A \sum_{s} D_{s} \sin \frac{2 \pi s m}{M}\right]\right\} \\
& +\frac{1}{2 N a}-\sum_{m=1}^{N} \sum_{n} V_{n}^{\prime} \cos \left\{\frac{2 \pi n}{b}\left[\left(m+\frac{1}{2}\right) a-A \sum_{a} D_{s} \sin \frac{2 \pi s m}{N}\right]\right\}+\frac{\pi A}{4 N a} \sum_{s} s D_{s}^{2}
\end{aligned}
$$

where we have written $A$ for $\left[(1 / \lambda)+\left(1 / \lambda^{\prime}\right)\right]$.

${ }^{4}$ I. S. Sokolnikoff, Malhematical Theory of Elasticity (McGraw-Hill Book Company, Inc., New York, 1956), p. 342. 
Because (15) is a variational expression, an upper bound can be found to the energy by making an arbitrary choice of the variational parameters $D_{s}$. Useful information can be obtained in this way by the choice

$$
D_{0}=N a \epsilon / A=M b \epsilon / A ; \quad D_{s}=0, s \geqslant 1,
$$

corresponding to a misfit interface with no elastic strain, which gives

$$
\begin{aligned}
E^{\prime}= & \frac{1}{2 M b} \sum_{m=1}^{M} \sum_{n} V_{n} \cos \left\{\frac{2 \pi n}{a}\left[\left(m+\frac{1}{2}\right) b+N a \epsilon\right]\right\} \\
& +\frac{1}{2 N a} \sum_{m=1}^{N} \sum_{n} V_{n}{ }^{\prime} \cos \left\{\frac{2 \pi n}{b}\left[\left(m+\frac{1}{2}\right) a-M b \epsilon\right]\right\} .
\end{aligned}
$$

On summing over $m$ as indicated, the only terms remaining in the first summation are those for which

$$
n b / a \equiv n N / M=k,
$$

where $k$ is an integer. Each such $m$ term contributes $V_{n} \cos \pi(k+2 M k \epsilon)$ to the sum. A similar result applies for the second summation when

$$
n a / b \equiv n M / N=k
$$

and, using (13), the contribution of each $m$ term is $V_{n}^{\prime} \cos \pi(k+2 N k \epsilon)$. Since $N$ and $M$ have no common factor the resonance conditions (18) and (19) exclude all terms except those for which $n=M k$ or $n=N k$. Thus

$$
\begin{aligned}
E^{\prime}=\frac{1}{2 b} \sum_{k} V_{M k} \cos \pi & (k+2 M k \epsilon) \\
& +\frac{1}{2 a} \sum_{k} V_{N k^{\prime}} \cos \pi(k+2 N k \epsilon) .
\end{aligned}
$$

Simple minimization with respect to $\epsilon$ then yields a best value for $E^{\prime}$.

This result already provides useful information. For most physical situations the Fourier components $V_{n}$ and $V_{n}{ }^{\prime}$ of the surface potential decrease rapidly after the first few terms. Equation (20), therefore, shows that the interfacial energy has minima when either $N$ or $M$ or both are small integers. We thus conclude that the misfit energy has minima when the lattice constants of the two crystals are in simple ratio.

The choice of variational parameters made in (16) corresponds to an interface with no elastic strain. For a more general result in the regions not too close to these primary resonance minima we suppose that the displacement amplitudes $A D_{s}$ are small so that trigonometric. functions depending on them can be approximated by the first term in their series expansion. Then (15) can be written, after some manipulation, in the approximate form

$$
\begin{array}{rl}
E \approx E^{\prime}-\frac{\pi A}{2 M a b} \sum_{n} \sum_{s} & n V_{n} D_{s} \sum_{m=1}^{M}\{\cos [2 \pi m(n N-s) / M]-\cos [2 \pi m(n N+s) / M]\} \\
& +\frac{\pi A}{2 N a b} \sum_{n} \sum_{s} n V_{n}^{\prime} D_{s} \sum_{m=1}^{N}\{\cos [2 \pi m(n M-s) / N]-\cos [2 \pi m(n M+s) / N]\}+\frac{\pi A}{4 N a} \sum_{s} s D_{s}{ }^{2} .
\end{array}
$$

The elastic distortion term involving $D_{s}$ is only able to lower the energy $E$ when one of the subsidiary resonance conditions

or

$$
\left.\begin{array}{l}
(n N \pm s) / M=k \\
(n M \pm s) / N=k
\end{array}\right\}
$$

is fulfilled, $k$ being an integer. From the physics of the problem, since the lattice consists of discrete atoms $s$ is restricted to be less than $N$ or $M$, respectively, in these two expressions. Thus either

$$
|k M \pm N| / n N<1 \text { or }|k N \pm M| / n M<1,
$$

respectively. When the relevant one of (22) is satisfied summation over $m$ gives a nonzero result and

$$
\begin{gathered}
E \approx E^{\prime} \pm \frac{\pi A}{2 a b} \sum_{n} \sum_{k}^{\prime}\left[n V_{n} D_{|k M-n N|}-n V_{n}^{\prime} D_{|k N-n, M|}\right] \\
+\frac{\pi \cdot A}{4 N a} \sum_{n} \sum_{k}^{\prime}\left[|k M-n N| D_{|k M-n N|^{2}}\right. \\
\left.+k N-n M D_{|k N-n M|^{2}}\right]
\end{gathered}
$$

the $k$ summations being restricted so that (23) are satisfied. This expression can now be minimized with respect to the various $D_{s}$, giving

$$
D_{|k M-n N|}=\mp\left(V_{n} / b\right)(n N /|k M-n N|)
$$

with a similar result for $D_{|k N \sim n M|}$, and

$$
\begin{aligned}
E \approx E^{\prime}-\frac{\pi A}{4 a b} \sum_{k}^{\prime} \sum_{n}\left[\frac{n}{b} V_{n}^{2} \frac{n N}{|k M-n N|}\right. & \\
& \left.+\frac{n}{a} V_{n}{ }^{2} \frac{n M}{|k N-n M|}\right]
\end{aligned}
$$

From (26) it is clear that the double sum has energy minima whenever $|k M-n N|$ or $|k N-n M|$ vanish. These minima occur for the same $M / N$ ratios as do the minima in $E^{\prime}$. It is no longer true, however, that (26) represents an upper bound to the energy since (21) was derived on the assumption that $A D_{s}$ was small compared to $a$ or $b$ and this is clearly not satisfied near a resonance, as seen from (25). The result (26) is, 


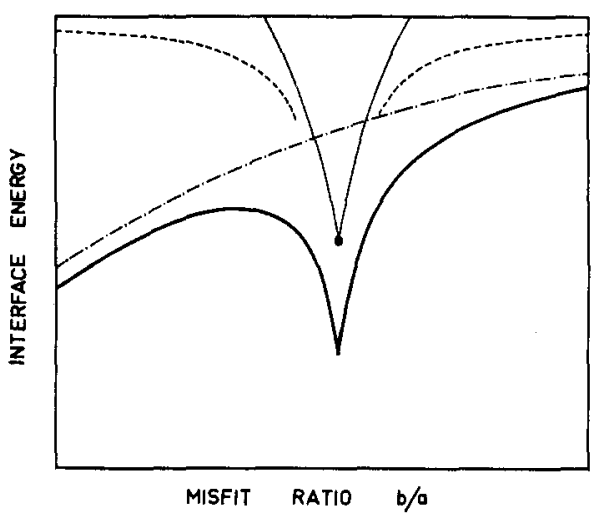

FIG. 1. Various approximations and their summation in calculation of interface energy near a resonance minimum. $-E^{\prime}$ from $(20) ;-C E$ from $(33) ;-.-E$ from $(26)$ with resonant term omitted; $--\cdots E$ due to resonant term in (26); - best approximation to total energy.

however, a valid approximation as an upper bound, except close to major resonances, providing any $D_{s}$ which are of large value are simply arbitrarily set equal to zero.

Very near to a major resonance a different approach must be adopted. Physically, near the fundamental $(1,1)$ resonance, we expect to be able to describe the interface in terms of a dislocation model-large regions of almost perfect fit separated by small regions, the dislocations, in which the fit is very bad. A variational model may be based on this picture for the more general resonances which are considered here.

Suppose that the first of the conditions (22) is satisfied with $N$ and $M$ large and $s=s_{0}$, a small integer, corresponding to a condition near resonance. Referring to (15), let us choose the $D_{s}$ in such a way that

$$
A \sum_{s} D_{s} \sin -\frac{2 \pi s m}{M}=\frac{a s_{0} m}{n M}+N a \epsilon
$$

in the region $-M / 2 s_{0}<m<M / 2 s_{0}$, with similar "sawtooth" behavior in neighboring regions. Clearly (27) involves only terms with $s$ a multiple of the fundamental $s_{0}$. The assumption (27) leads to a behavior in which the two lattices are coherent over most of the interface, with dislocations spaced at equal distances $M b / s_{0}$.

Substituting (27) into (15), using (13) to simplify the second summation, and comparing the result with (17) we find

$$
E \approx E_{R}{ }^{\prime}+\frac{s_{0} E^{\prime \prime}}{N a}+\frac{\pi A}{4 N a} \sum_{t} s_{0} t D_{s_{0} t^{2}}{ }^{2},
$$

where $E_{R}{ }^{\prime}$ is the value of $E^{\prime}$ exactly at the resonance considered and $E^{\prime \prime}$ is the misfit energy of a single dislocation. The last term takes account of the elastic strain energy associated with the dislocations. Since each dislocation involves misfit over only a spacing $a / n$ or $b / k$, an upper bound to $E^{\prime \prime}$ is given approximately by

$$
E^{\prime \prime} \lesssim-2 E_{R}^{\prime} a / n
$$

Now to satisfy (27) we require

$$
D_{0}=N a \epsilon / A ; \quad D_{l s_{0}}=(-1)^{t+1} a / n A l \pi
$$

so that the series in (28) diverges. However it is legitimate to truncate this series so that

$$
t<M k / 2 s_{0} \approx n N / 2|k M-n N|
$$

since the dislocation is not localized to less than a distance $b / k$. An approximate value for this truncated series for moderately large $T$ is

$$
\sum_{t=1}^{T} t^{-1} \approx \ln (3 T / 2)
$$

so that we can write finally

$$
\begin{aligned}
& E \approx E_{R}{ }^{\prime}+\frac{|k M-n N|}{n N} \\
& \quad \times\left[\frac{a}{4 \pi n A} \ln \left(\frac{3 n N}{4|k M-n N|}\right)-2 E_{R^{\prime}}\right] .
\end{aligned}
$$

This value of $E$ is also an upper bound to the interfacial energy.

It can be seen from (33) that $E$ reduces simply to $E_{R}{ }^{\prime}$ as an upper bound at the resonance in agreement with (20). For lattice ratios departing only slightly from resonance (33) implies an approximately linear increase of interfacial energy with the misfit, $|k M-n N| / n N$. This is to be expected since the number of dislocations increases linearly with misfit and, for large dislocation spacings, the interaction between the dislocation strain fields is small.

With the aid of the approximate results and upper bounds (20), (26), and (33), a reasonable approximation to the interfacial energy at any degree of misfit can be constructed. The expression (33), together with (20), gives an upper limit to energy near a major resonance, and a good approximation to the energy can be obtained by adding the "background" contribution from (26) with the major resonance term omitted. In the wings of the resonance minimum the complete expression (26) is a better approximation to the energy. This procedure is illustrated schematically in Fig. 1 for the case of a moderately prominent resonance minimum.

\section{SPECIFIC EXAMPLES}

It is helpful now to consider the one-dimensional case for two specific assumed potentials - sinusoidal and parabolic - both of which have been discussed by other authors. ${ }^{2,3}$ The sinusoidal potential is particularly simple, having only one Fourier component, while the parabolic potential is a physically reasonable example of 
a potential with a complete set of Fourier components. In the course of the calculation we assume that the lattice parameter $a$ is kept fixed while $b$ is varied.

Some additional assumption must be made about the elastic moduli $\lambda$ and $\lambda^{\prime}$. They might conveniently be assigned arbitrary values, but it is perhaps more reasonable physically to relate them to the potentials $V$ and $V^{\prime}$. We make the assumptions that the interactions between atoms of both crystals are similar so that $V_{n}{ }^{\prime}=V_{n}$, and that the atoms of each crystal are arranged on simple close-packed lattices with parameters $a$ and $b$, respectively. Consideration of small shear distortions then shows that

$$
\lambda a^{2}=\lambda^{\prime} b^{2}=-4 \pi^{2} B \sum_{n} n^{2} V_{n}
$$

where $B$ is a constant of order unity which we arbitrarily set equal to 2 for this calculation.

For the sinusoidal potential we let

so that

$$
V_{1}^{+}=-V_{n}=0, \quad n \neq 1,
$$

$$
A=\left(a^{2}+b^{2}\right) / 8 \pi^{2} V .
$$

The expression (20) for $E^{\prime}$ is easily minimized with $\epsilon=\frac{1}{2}$ giving

$$
\begin{aligned}
& E^{\prime}=-V / a, \quad b=a ; \quad E^{\prime}=-r / 2 k a, \quad b=k a ; \\
& E^{\prime}=-V / 2 a, \quad a=k b .
\end{aligned}
$$

The approximations (26) and (33) are readily calculated and the full result is shown in Fig. 2. It can be seen that the behavior of interfacial energy near the fundamental $(1,1)$ resonance is very similar to that calculated by van der Merwe,, 3 but that important subsidiary minima occur at $b=a / 2$ and $b=2 a$. The asymmetry between these two resonances is due to the fact that $b$ is varied, and with it the elastic constant $\lambda^{\prime}$, while $a$ is held fixed.

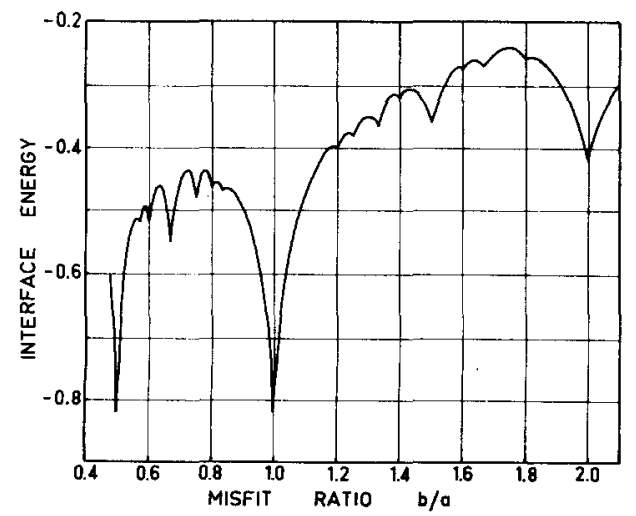

Frg. 2. Interface energy in units of $V / a$ for a sinusoidal potential of amplitude $V$. The lattice spacing $a$ remains constant while $b$ is varied. Elastic coefficients vary with lattice spacing under the assumption that the interaction potential $V$ remains constant.

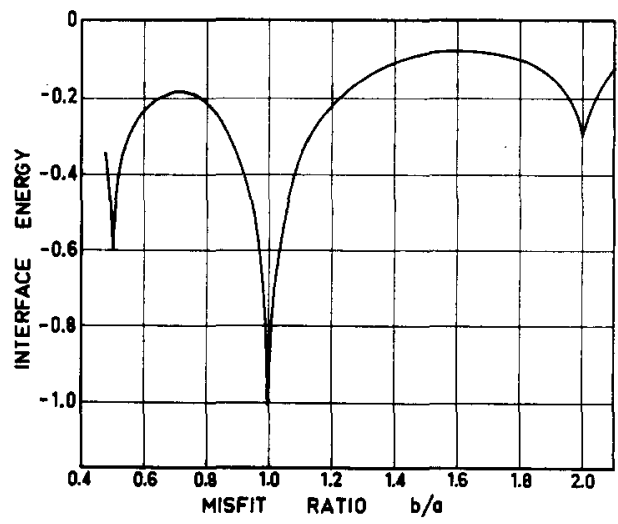

FIG. 3. Interface energy in units of $V / a$ for a parabolic potential, the amplitude of whose first Fourier component is $V$. The lattice spacing $a$ remains constant while $b$ is varied. Elastic coefficients vary with lattice spacing under the assumption that the interaction potential $V$ remains constant. The subsidiary minima in the interface energy occur for lattice spacings which are in the ratio of two small integers.

In the case of a parabolic potential all Fourier components exist and we can write

$$
V_{0}=0 ; \quad V_{n}=(-1)^{n} n^{-2} V, \quad n \geqslant 1 .
$$

The series (34) does not converge, but direct calculation gives

$$
A=\left(a^{2}+b^{2}\right) / 4 \pi^{2} V .
$$

The value of $\epsilon$ required to minimize (20) is no longer obvious but the choice $\epsilon=\frac{1}{2}$ again appears to minimize $E^{\prime}$ and certainly yields a valid upper bound. With this choice $E^{\prime}$ can be evaluated in terms of a Riemann zeta function, ${ }^{5}$ giving

$$
E^{\prime}=\frac{V}{2 a} \frac{(M+N)}{N^{2} M} \sum_{k}(-1)^{k} k^{-2}=-0.411 \frac{(M+N)}{N^{2} M} \frac{V}{a}
$$

from which it is clear that energy minima exist whenever both $N$ and $M$ are small integers. The approximations (26) and (33) are again readily calculated and the result is shown in Fig. 3.

Examination of this figure shows that the subsidiary minima, which are missed by the analysis of van der Merwe, form an important part of the complete picture. The interface energy has a more complicated behavior than for a sinusoidal potential because of resonances involving higher Fourier components of the potential. The resonance minima are broader than for the sinusoidal case because of the smaller values of the elastic parameters $\lambda$ and $\lambda^{\prime}$.

\section{DISCUSSION}

The variational approach which has been presented allows energy calculations to be made for a wide variety of interface problems. A particular virtue of the approach is that it takes account automatically both

${ }^{5}$ E. Jahnke and F. Emde, Tables of Functions (Dover Publications, Inc., New York, 1945), pp. 269-274. 
of crystal symmetry and of the detailed form of the surface potential.

Application of the variational method to simple one-dimensional problems shows that by its use many finer details of interface behavior, such as the subsidiary minima for parabolic potentials, are clearly brought out. The present theory would also take account of symmetry effects in calculation of the energy of the interface between two three-dimensional crystals rotated relative to one another about an axis normal to the interface and give a set of cusped energy minima. A related two-dimensional problem was in fact considered some time ago from the viewpoint of a dislocation model by Read and Shockley ${ }^{6}$ who discussed also the existence of cusped energy minima at particular orientations. These minima are of importance in the theory of oriented crystal overgrowths.

From the form of (33) it can be seen that the width of an individual resonance minimum is proportional to the elastic parameter $A$, that is, to $\left(\lambda+\lambda^{\prime}\right) / \lambda \lambda^{\prime}$. Thus if one of the crystals has a very small shear modulus the width of the peaks becomes very great and the over-all interfacial energy is lowered.

${ }^{6}$ W. 'T. Read and W. Shockley, Phys. Rev. 78, 275 (1950).
It is possible, with little complication, to exten analysis to cover cases where the thickness of one both crystals is finite. This extension follows the method of van der $\mathrm{Merwe}^{2}$ and involves simply a modification to the form of the elastic strain terms in (9) and (10). If the thickness of each crystal is greater than a characteristic length of the superlattice then the correction terms are negligible. For crystals of smaller thickness the interfacial energy is reduced by the correction terms.

Finally it should be noted that in many problems it is the free energy rather than simply the energy of the interface which is important. This is always a consideration in systems at a $f$ ite temperature, but for "ordinary" materials wellbelow their melting points the correction is not usually large. In some highly bonded molecular crystals of unusual structure however, such as ice, the configurational entropy term may be of considerable importance if the other crystal of the pair tends to impose molecular orientations at the interface. ${ }^{7}$ In such a case the entropy contribution to the free energy must be added to the energy term which has been the subject of the present calculation.

\title{
Mechanism of Gold Diffusion into Silicon
}

\author{
W. R. Wricox \\ Aerospace Corporation, El Segundo, California \\ AND \\ T. J. LaChapel.te \\ Pacifuc Semiconductors, Incorporated, Lawendale, California
}

(Received 14 June 1963)

\begin{abstract}
Gold was found to diffuse into silicon by a complex mechanism involving a vacancy-controlled interstitialsubstitutional equilibrium. This led to very complex diffusion concentration profiles. In analyzing the problem, a new experimental value was found for the self-diffusion coefficient of silicon, $1.81 \times 10^{4}$ $\exp (-112 \pm 20 \mathrm{kcal} / R T) \mathrm{cm}^{2} / \mathrm{sec}$, which compared very favorably with previous data on diffusion of bismuth, germanium, and tin in silicon. The interstitial gold-diffusion coefficient was found to be $2.4 \times 10^{-4}$ $\exp (-8.9 \pm 2 \mathrm{kcal} / R T)$, the substitutional gold-diffusion coefficient to be $2.75 \times 10^{-3} \exp (-47 \pm 10 \mathrm{kcal} / R T)$, the equilibrium-interstitial gold solubility to be $5.95 \times 10^{24} \exp (-58 \pm 10 \mathrm{kcal} / R T)$, and the equilibriumsubstitutional gold solubility (Collin's data) below $1200^{\circ} \mathrm{C}$ to be $8.15 \times 10^{22} \exp (-40.6 \mathrm{kcal} / R T)$.
\end{abstract}

\section{INTRODUCTION}

G OLD conveniently furnishes recombination centers in silicon. ${ }^{1-5}$ Because of this, gold has been deliberately diffused into silicon to make high-speed

${ }^{1}$ G. Bemski, Phys. Rev. 111, 1515 (1958).

2 R. O. Carlson, J. Appl. Phys. 29, 1001 (1958).

${ }^{3}$ C. B. Collins, R. O. Carlson, and C. J. Gallagher, Phys. Rev. $105,1168(1957)$.

${ }^{4}$ W. D. Davis, Phys. Rev. 114, 1006 (1959).

${ }^{5}$ A. E. Bakanowski and J. H. Forster, Bell System Tech. J. $39,87(1960)$. semiconductor devices. The diffusion of gold into silicon was first studied in detail by Struthers using radiotracers. ${ }^{6}$ The diffusion coefficients measured gave a good Arrhenius plot from $800^{\circ}$ to $1200^{\circ} \mathrm{C}$ to yield $D=0.011$ $\times \exp (-25800 / R T)$. More recent work, however, has indicated that gold diffuses into silicon at a much slower rate. ${ }^{7.8}$ Limited evidence that the diffusion may be

\footnotetext{
6 J. D. Struthers, J. Appl. Phys. 27, 1560 (1956); 28, 516 (1957).

${ }^{7}$ B. I. Boltaks, G. S. Kulikov, and R. S. Malkovich, Fiz.
} 